\title{
Über die Beziehung zwischen der Haupt= und Mitagglutination.
}

IX. Mitteilung.

Beobachlung über die Mitagglutination von Typhus- und Paratyphus

B-Bazillen während der Immunisierung von Kaniuchen mit Paratyphus A-Bazillen.

Von

Prof. Dr. Kaoru Aoki u. Dr. Tsunetaro Konno.

(青木䕒) (昆野恒太郎)

(Aus dem bakteriologischen Institute der Toloku Universität $z u$ Sendai.)

Wir hatten schon in der ersten, dritten, sechsten und achten Mitteilung angegeben, dass, wenn Kaninchen lange Zeit andauernd mit Typhusbazillen vorbehandelt werden, Paratyphus B-Bazillen gegenüber, wenn mit Paratyphus B-Bazillen immunisiert, Typhusbazillen und Paratyphus A-Bazillen gegenüber, wenn mit Aerthryck bazillen immunisiert, Paratyphus B-Bazillen gegenüber es nachweisbar ist, dass der Wert des Bruches, welcher die Beziehung zwischen der Haupt- und Mitagglutination auf die Weise ausdrückt, dass der Titer der Mitagglutination mit dem Titer der Hauptagglutination dividiert wird, während der ganzen Immunisierungszeit eine bestimmte Schwankung zeigt, nämlich im Anfang der Immunisierung grösser, dann kleiner und schliesslich wieder grösser wird, weil die Hauptagglutination nicht nur für sich allein, sondern auch im Vergleich mit der Mitagglutination, im ersten Stadium der Immunisierung mit grösserer Geschwindigkeit und in ihrem letzteren Stadium mit kleinerer Geschwindigkeit, dagegen die Mitagglutination, nicht nur für sich allein, sondern auch im Vergleich mit der Hauptagglutination, im ersten Stadium der Immunisierung in geringerem und in ihrem letzteren Stadium in höherem Grade sich zu vermehren pflegt. Der Krümmungsgrad der Kurvenlinie, welche diese Schwankung des Wertes des Bruches während 
der ganzen Vorbehandlungen darstellt, gestaltet sich dabei sehr verschieden, je nach der verwandtschaftlichen Beziehung zwischen der betreffenden Bakterienarten, je nach der Weise der Immunisierung und je nach der Individualität der Tiere.

Hier beabsichtigen wir, zu untersuchen, ob diese Erscheinung bei der Immunisierung von Kaninchen mit Paratyphus A-Bazillen Typhusbazillen und Paratyphns B-Bazazillen gegenüber zum Vorschein kommt.

\section{Subkutane Immunisierung.}

\section{Versuch 1.}

Sechs Kaninchen wurden mit unten angegebenen Dosen von Paratyphus-A-Bazillen im ganzen neunmal in siebentägigen Intervallen vorbehandelt. Die Dosen waren $\frac{1}{1000}, \frac{1}{100}, \frac{1}{10}, \frac{1}{5}, \frac{1}{2}, 1,2,3$ und 4 Agarkulturen. An jedem siebenten Tage nach der Einspritzung wurde eine Blutprobe entnommen und auf ihren Titer der Hauptagglutination und Mitagglutination gegen Typhus- und Paratyphus B-Bazillen geprüft. Der Titer der Agglutination von den einzelnen Tieren wurde durchschnittlich berechnet. Aus dieser Zabl wurde der Wert des Bruches berechnet, welcher die Beziehung zwischen der Haupt-und Mitagglutination auf die Weise ausdrückt, duss der Titer der Mitagglutination mit dem Titer der Hauptagglutination disidiert wird. Ferner wurde die Vermehrungsgeschwindigkeit der beiden Reaktionen auf die Weise beobachtet, dass der Titer der Agglutination bei der betreffenden Vorbehandlung mit den 'Titer derselben bei der vorangehenden Vorbehandlung dividiert wird.

\section{Versuch 2.}

Derselbe Versuch wurde wit noch grösseren Dosen derselben Bakterien bei sechs Kaninchen ausgeführt. Die Dosen waren $\frac{1}{10}, \frac{1}{5}, \frac{1}{2}, 1,2,3,4,5,6$ u. 7 Agarkulturen. Deshalb wurden die Tiere im ganzen zehnmal vorbehandelt. Die Resultate wurden auf die gleiche Weise behandelt und beobachtet, wie im vorigen Versuche.

\section{Versuch 3.}

Mit noch grösseren Dosen von denselben B.kterien wurde derselbe Versuch bei sechs Kaninchen ausgeführt. Die Dosen waren $\frac{1}{2}, 1,2,3,4,5,6$ u. 7 Agarkulturen. Die Tiere wurden im ganzen achtmal vorbehandelt. Die Resultate wurden auf die gleiche Weise behandelt und beobachtet, wie im vorigen Versuche.

Die Resultate aus diesen drei Versuchen wurden je nach den Bakterienarten, mit denen die Mitagglutination ausgeführt wurde, angeordnet und betrachtet. Zuerst wurden sie nämlich nach Paratyphus B-Bazillen angeordnet, so dass man dadurch eine Tabelle erhielt (Tab. 1). Aus dieser Tabelle war folgendes ersichtlich: Die Vermehrungsgeschwindigkeit trat im ersten Versuche bei der Hauptagglutination bei den ersten fünf Vorbehandlungen bedeutend stärker als bei der Mitagglutination ein, welche zu dieser Zeit auch für sich 
allein stark zugenommen hatte. Bei den sechsten und siebenten Immunisierungen zeigte sie sich bei der vorderen etwas schwächer und bei den achten und neunten Immunisierungen wieder etwas stärker als bei der letzteren. Infolgedessen zeigte sich der Wert des Bruches zuerst gross, dann kleiner und endlich wieder grösser. Bei dem zweiten Versuche zeigte die Hauptagglutination ebenfalls im Anfang der Im-

Tabelle 1.

\begin{tabular}{|c|c|c|c|c|c|}
\hline & \multirow{2}{*}{$\begin{array}{l}\text { Mal u. Dose } \\
\text { der } \\
\text { Einspritzung }\end{array}$} & \multirow{2}{*}{$\begin{array}{l}\text { Beziehung } \\
\text { zwischen der } \\
\text { Haupt-u. Mit } \\
\text { agglutination }\end{array}$} & \multirow{2}{*}{$\begin{array}{c}\text { Mal der } \\
\text { Vorbehandlung }\end{array}$} & \multicolumn{2}{|c|}{$\begin{array}{l}\text { Vermehrungsverbalten der } \\
\text { Haupt- und Mitagglutination }\end{array}$} \\
\hline & & & & Hauptaggl. & Mitaggl. \\
\hline$\frac{\tilde{E}}{3}$ & $\begin{array}{l}\text { I } \frac{1}{1,000} \text { Agar } \\
\text { II } \frac{1}{100} \\
\text { III } \frac{1}{10} \\
\text { IV } \frac{\frac{1}{5}}{\text { V } \frac{1}{2}} \\
\text { VI } 1 \\
\text { VII } 2 \\
\text { VIII } 3 \\
\text { IX } 4\end{array}$ & $\begin{aligned} \frac{35}{250} & =\frac{1}{7} \\
\frac{53}{433} & =\frac{1}{8} \\
\frac{58}{3,333} & =\frac{1}{57} \\
\frac{50}{7,116} & =\frac{1}{142} \\
\frac{50}{12,500} & =\frac{1}{250} \\
\frac{58}{13,333} & =\frac{1}{229} \\
\frac{66}{14,166} & =\frac{1}{214} \\
\frac{66}{15,833} & =\frac{1}{239} \\
\frac{50}{20,000} & =\frac{1}{400}\end{aligned}$ & $\begin{array}{r}I-I I \\
I I-I I I \\
I I I-I V \\
I V-V \\
V-V I \\
V I-V I I \\
V I I-V I I I\end{array}$ & $\begin{array}{l}\frac{433}{250}=1,7 \\
\frac{3,333}{433}=7,7 \\
\frac{7,116}{3,333}=2,1 \\
\frac{12,500}{7,116}=1,7 \\
\frac{13,333}{12,500}=1,0 \\
\frac{14,166}{13,333}=1,0 \\
\frac{15,833}{14,166}=1,1 \\
\frac{20,000}{15,833}=1,2\end{array}$ & $\begin{array}{l}\frac{53}{35}=1,5 \\
\frac{58}{53}=1,0 \\
\frac{50}{58}=0,8 \\
\frac{50}{50}=1,0 \\
\frac{58}{50}=1,1 \\
\frac{66}{58}=1,1 \\
\frac{66}{66}=1,0 \\
\frac{50}{66}=0,7\end{array}$ \\
\hline 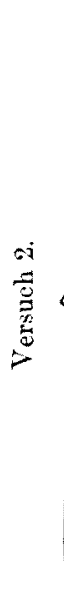 & $\begin{aligned} \text { I } & \frac{1}{10} \text { Agar } \\
\text { II } & \frac{1}{5} \\
\text { III } & \frac{1}{2} \\
\text { IV } & 1 \\
\text { V } & 2 \\
\text { VI } & 3 \\
\text { VII } & 4 \\
\text { VIII } & 5 \\
\text { IX } & 6 \\
\text { X } & 7\end{aligned}$ & 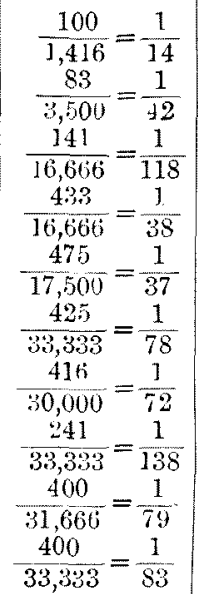 & $\begin{array}{r}I-I I \\
I I-I I I \\
I I-I V \\
I V-V \\
V-V I \\
V I-V I I \\
V I I-V I I I \\
V I I I-I X \\
I X-X\end{array}$ & $\begin{array}{l}\frac{3,500}{1,416}=2,4 \\
\frac{16,666}{3,500}=4,7 \\
\frac{16,666}{16,666}=1,0 \\
\frac{17,500}{16,666}=1,0 \\
\frac{33,333}{17,500}=1,9 \\
\frac{30,000}{33,000}=0,9 \\
\frac{38,000}{30,000}=1,1 \\
\frac{31,666}{33,33: 3}=0,9 \\
\frac{33,333}{31,666}=1,0\end{array}$ & $\begin{array}{l}\frac{83}{100}=0,8 \\
\frac{141}{83}=1,7 \\
\frac{433}{141}=3,0 \\
\frac{475}{433}=1,0 \\
\frac{425}{475}=0,9 \\
416 \\
425=0,9 \\
241 \\
416=0,5 \\
400 \\
241=1,6 \\
400 \\
400=1,0\end{array}$ \\
\hline
\end{tabular}




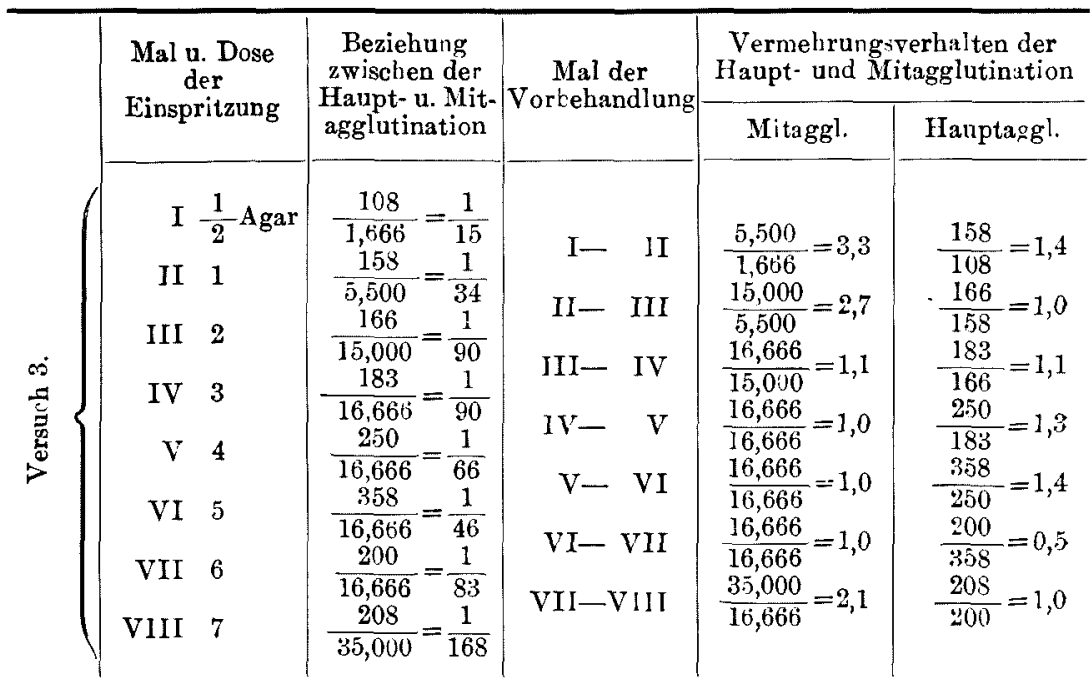

munisierung, und zwar in den ersten drei Vorbehandlungen, eine stärkere Vermehrungsgeschwindigkeit, nicht nur für sich allein, sondern anch im Vergleich zu der Mitagglutination. Bei der vierten Vorbehandlung trat die Vermehrungsgeschwindigkeit bei der Hauptagglutination schwächer als bei der Mitagglutination ein. Deshalb zeigte sich der Wert des Bruches, welcher von der ersten Vorbehandlung allmählich immer kleiner, nämlich von $\frac{1}{14}$ bis $\mathrm{zu}_{11 \overline{8}}^{1}$ wurde, von der vierten Vorbehandlung plötzlich grösser, nämlich $\underset{\mathbf{3 8}}{\mathbf{1}}$. Dieser Wert fing an, von der sechten Immunisierung ab wieder kleiner zu werden, so dass er bei der achten Vorbehandlung $\frac{1}{13 \overline{8}}$ wurde, weil die vormehrungsgeschwindigkeit bei der Hauptagglutination von der sechsten Einspritzung viel grösser als bei der Mitagghutination war. Bei der neunten und zehnten Vorbehandlung nahm er wieder zu. Aus dem dritten Versuche ist auch zu ersehen, dass die Vermehrungsgeschwindigkeit in den ersten drei Vorbehandlungen bei der Hauptagglutination viel stärker als bei der Mitagglutination und in der vierten Vorbehandlung bei ersterer gerade so stark wie bei letzterer eintrat. Deshalb wurde der Wert des Bruches von der ersten Einspritzung bis zur dritten, eventuell bis zur vierten Vorbehandlung immer kleiner, nämlich von $\frac{1}{15}$ bis $\frac{1}{90}$. Dieser wert nahm bei der fünften und sechsten Vorbehandlung etwas zu; er betrug nämlich $\frac{1}{46}$, weil die Vermehrungsgeschwindigkeit bei diesen Vorbehandlungen bei der Hauptagglutination kleiner als bei der Mitagglutination war. Von der siebenten 
Tabelle 2.

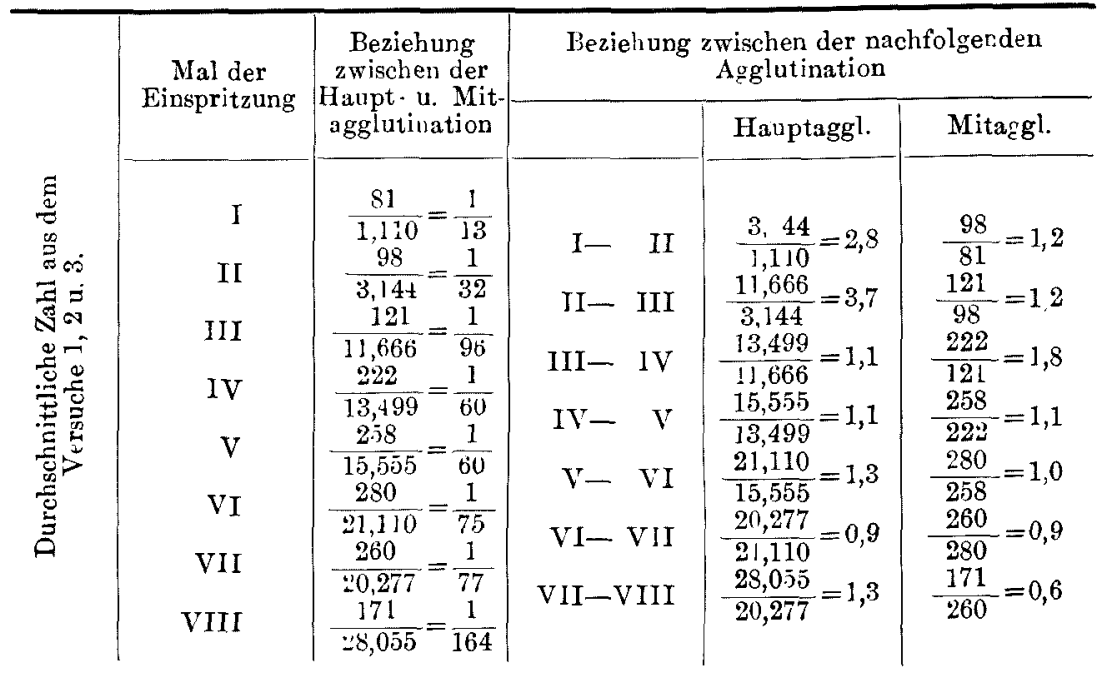

Vorbehandlung zeigte sie sich bei ersterer viel grösser als bei letzterer, so dass der Wert des Bruches sich wieder kleiner, nämlich $\frac{1}{16 \overline{8}}$, darstellte. Nach diesen Ergebnissen ist es klar, dass die Erscheinung, dass der Wert des Bruches, welcher die Beziehung zwischen der Haupt- und Mitagglutination darstellt, im Anfang der Immunisierung gross, dann kleiner und endlich wieder grösser wird, wie schon bei den anderen Bakterien nachgewiesen war, hier bei allen Versuchen, besonders bei dem zweiten und dritten Versuche deutlich nachgewiesen wurde. Wenn man aber eine durchschnittliche Zahl aus dieser Tabelle ausrechnete, wie es Tabelle 2 darstellt, so trat hier auch die oben auseinandergesetzte Erscheinung deutlich ein (Tab. 2). Die Vermehrungsgeschwindigkeit der Hauptagglutination zeigte sich in den ersten drei Vorbehandlungen viel stärker als die der Mitagglutination, welche in dieser Zeit auch stark zugenommen hatte. Deshalb wurde der Wert des Bruches, welcher bei der ersten Immunisierung $\frac{1}{\mathbf{1 3}}$ gross war, bei der zweiten Immunisierung $\frac{1}{32}$ und bei der dritten Vorbehandlung $\frac{1}{96}$ gross. Dieser Wert wurde von der vierten und fünften Immunisierung ab wieder grösser, nämlich $\frac{\mathbf{x}}{60}$, weil die Vermehrungsgeschwindigkeit in dieser Zeit bei der Hauptagglutination kleiner als bei der Mitagglutination war. Dieser Wert fing an, mit weiteren Immunisierungen allmählich wieder lekiner zu werden, so dass er endlich bei der achten Immunisierung 
$\frac{1}{164}$ wurde, weil die Hauptagglutination in diesem Stadium mit stärker und die Mitagglutination mit schwächerer Geschwindigkeit zunahm. Den Verlauf des Wertes kann man in einer Kurvenlinie auf die Weise darstellen, dass der Nenner des Bruches als die Ordinate, das Mal der Vorbehandlung als die Abszisse betrachtet wird, wie Kurve 1 zeigt.

Kurve 1.

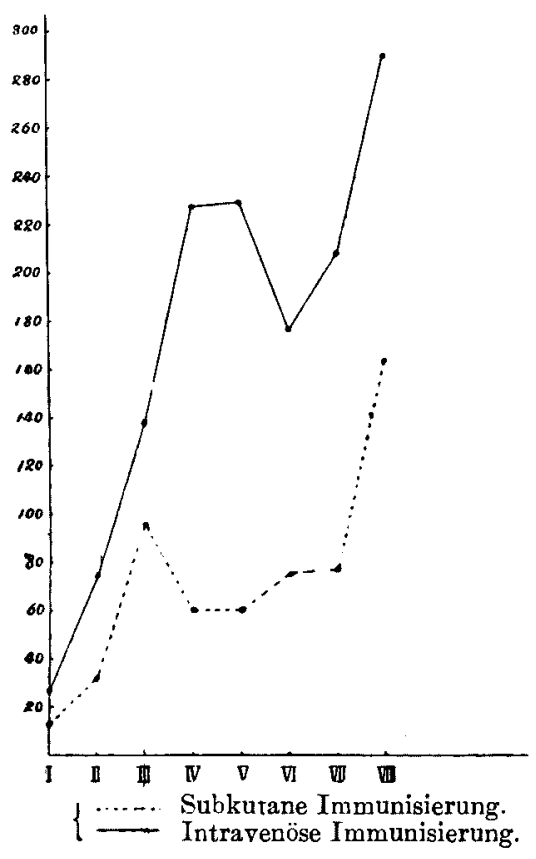

Wenn ferner die Resultate aus den drei Versuchen auf die Mitagglutination von Typhusbazillen hin betrachtet wurden, so erhielten wir wieder eine andere Tabelle, nämlich Tab. 3. Darau kann man folgendes ersehen: Bei dem Versuch 1 findet man, dass die Vermehrungsgeschwindjgkeit in den ersten vier Immunisierungen bei der Hauptagglutination bedeutend grösser als bei der Mitagglutination und ferner in der fünften und sechsten Vorbehandlung umgekehrt bei ersterer schwächer als bei der letzterer eintrat. Infolgedessen zeigte sich der Wert des Bruches, welcher bei der ersten Vorbehandlung $\frac{\mathbf{1}}{\mathbf{3 , 3}}$ gross war, von der zweiten Vorbehandlung immer kleiner, so dass er bei 
der vierten Vorbehandlung $\frac{1}{66}$ gross wurde. Er fing bald an, von der fünften Vorbehandlung zuzunehmen, so dass er bei der sechsten Immunisierung $\frac{1}{38}$ gross wurde. Dieser Wert des Bruches blieb in der siebenten Vorbehandlung fast unverändert gross, nahm bei der achten Vorbehandlung zu und bei der neunten Vorbehandlung wieder ab. Aus dem Versuch 2 ersieht man, dass die Vermehrungsgeschwindigkeit

Tabelle 3.

\begin{tabular}{|c|c|c|c|c|c|}
\hline & \multirow{2}{*}{$\begin{array}{l}\text { Mal u. Dose } \\
\text { der } \\
\text { Einspritzung }\end{array}$} & \multirow{2}{*}{$\begin{array}{c}\text { Beziehung } \\
\text { zwischen der } \\
\text { Haupt-u. Mit- } \\
\text { agglutination }\end{array}$} & \multirow{2}{*}{$\begin{array}{c}\text { Mal der } \\
\text { Vorbehandlung }\end{array}$} & \multicolumn{2}{|c|}{$\begin{array}{l}\text { Vermehrangsverhalten der } \\
\text { Haupt- und Mitagglutination }\end{array}$} \\
\hline & & & & Hauptaggl. & Mitaggl. \\
\hline 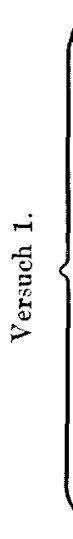 & $\begin{array}{l}\text { I } \frac{1}{1,000} \text { Agar } \\
\text { II } \frac{1}{100} \\
\text { III } \frac{1}{10} \\
\text { IV } \frac{1}{\frac{5}{5}} \\
\text { V } \frac{1}{2} \\
\text { VI } 1 \\
\text { VII } 2 \\
\text { VIII } 3 \\
\text { IX } 4\end{array}$ & $\begin{aligned} \frac{75}{250} & =\frac{1}{3,3} \\
\frac{83}{433} & =\frac{1}{5,2} \\
\frac{100}{3,333} & =\frac{1}{33} \\
\frac{108}{7,166} & =\frac{1}{66} \\
\frac{283}{12,500} & =\frac{1}{44} \\
\frac{350}{13,333} & =\frac{1}{38} \\
\frac{350}{14,166} & =\frac{1}{40} \\
\frac{616}{15,833} & =\frac{1}{25} \\
\frac{433}{20,000} & =\frac{1}{46}\end{aligned}$ & $\begin{array}{r}I-\text { II } \\
I I-I I I \\
I I I-I V \\
I V-V \\
V-V I \\
V I-V I I \\
V I I-V I I I \\
V I I J-J X\end{array}$ & $\begin{array}{l}\frac{433}{250}=1,7 \\
\frac{3,333}{433}=7,6 \\
\frac{7,166}{3,333}=2,1 \\
\frac{12,500}{7,166}=1,7 \\
\frac{13,333}{12,500}=1,0 \\
\frac{14,166}{13,303}=1,0 \\
\frac{15,833}{14,166}=1,1 \\
\frac{20,000}{15,5333}=1,2\end{array}$ & $\begin{array}{l}\frac{83}{75}=1,1 \\
\frac{100}{83}=1,2 \\
\frac{108}{100}=1,0 \\
\frac{283}{108}=2,6 \\
\frac{350}{28.3}=1,2 \\
\frac{350}{350}=1,0 \\
\frac{616}{350}=1,7 \\
\frac{433}{616}=0,7\end{array}$ \\
\hline 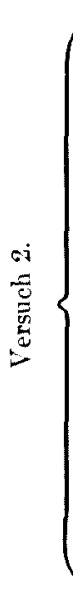 & $\begin{array}{rr}\text { I } & \frac{1}{10} \\
\text { II } & \frac{1}{5} \\
\text { III } & \frac{1}{2} \\
\text { IV } & 1 \\
\text { V } & 2 \\
\text { VI } & 3 \\
\text { VII } & 4 \\
\text { VIII } & 5\end{array}$ & $\begin{array}{l}\frac{250}{1,416}=\frac{1}{5,6} \\
\frac{216}{3,500}=\frac{1}{16} \\
\frac{450}{16,666}=\frac{1}{37} \\
\frac{616}{16,666}=\frac{1}{27} \\
\frac{533}{17,500}=\frac{1}{32} \\
\frac{666}{3:, 333}=\frac{1}{50} \\
\frac{833}{30,600}=\frac{1}{37} \\
\frac{833}{33,333}=\frac{1}{40} \\
\frac{916}{31,666}=\frac{1}{34} \\
\frac{1,166}{36,363}=\frac{1}{28}\end{array}$ & $\begin{array}{r}I-\text { II } \\
I I-\quad I I I \\
I I I-I V \\
I V-\quad V \\
V-V I \\
V I-V I I \\
V I I-V I I I \\
V I I I-I X \\
I X-X X\end{array}$ & $\begin{array}{l}\frac{3,500}{1,416}=2,4 \\
\frac{16,666}{3,500}=4,7 \\
\frac{16,666}{16,666}=1,0 \\
\frac{17,500}{16,666}=1,0 \\
\frac{33,333}{17,555}=1,9 \\
\frac{30,000}{33,333}=0,9 \\
\frac{33,383}{30,000}=1,1 \\
\frac{31,666}{33,33 ;}=0,9 \\
\frac{33,333}{31,6665}=1,0\end{array}$ & $\begin{array}{l}\frac{216}{250}=0,8 \\
\frac{450}{216}=2,0 \\
\frac{616}{450}=1,3 \\
\frac{533}{616}=0,8 \\
\frac{666}{533}=1,2 \\
\frac{833}{666}=1,2 \\
\frac{83: 3}{833}=1,0 \\
\frac{916}{833}=1,0 \\
\frac{1,166}{916}=1,2\end{array}$ \\
\hline
\end{tabular}




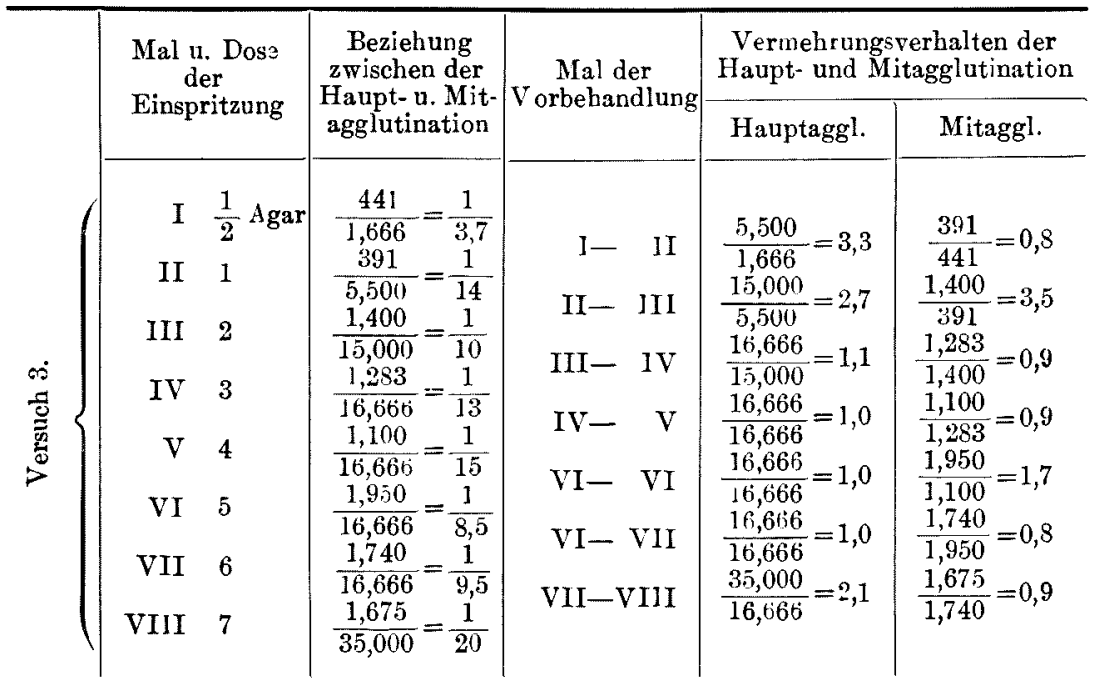

in der vorderen Immunisierung, nämlich in den ersten drei Vorbehandlungen bei der Hauptagglutination bedeutend stärker als bei der Mitagglutination, dagegen in der vierten Vorbehandlung bei ersterer viel schwächer als bei letzterer eintrat. Deshalb zeigte der Wert des Bruches, welcher bis zur dritten Vorbehandlung allmählich immer mehr abgenommen hatte, bei der vierten Vorbehandlung eine starke Zunahme. Dieser Wert des Bruches zeigte ferner in den darauffolgenden sechs Vorbehandlungen noch zweimalige Schwankungen, weil die Vermehrungsgeschwindigkeit bei den beiden Reaktionen bei jeder Einspritzung abwechselnd stärker und schwächer war. Bei dem Versuch 3 konnten wir die oben beschriebene Erscheinung nicht deutlich darstellen, weil der geringe Wechsel der $\mathrm{Zu}$ - und Abnahme der Vermehrungsgeschwindigkeit eintrat. Wenn aber eine durchschnittliche Zahl aus diesen Tabellen ausgerechnet wurde, wie es 'Tabelle 4 darstellt, so konnte man darin doch eine bestimmte Schwankung des Wertes des Bruches finden, wie oben auseinandergesetzt wurde. Die Vermehrungsgeschwindigkeit trat nämlich bei der Hauptagglutinatination in den ersten drei Vorbehandlungen bedentend stärker und in den nachfolgenden zwei Immunisierungen im minimalem Grade, aber immer stïrker als bei der Mitagglutination ein. Infolgedessen wurde der Wert des Bruches, welcher in der ersten Immunisierung $\underset{4,9}{\mathbf{1}}$ gross war, bis zur fünften Vorbehandlung $\frac{\mathbf{1}}{\mathbf{2}}$ klein. Von der sechsten Immunisierung fing er an zuzumehmen, so dass er in der siebenten Immunisierung $\frac{1}{20}$ 
Tabelle 4.

\begin{tabular}{c|c|c|c|c|c}
\hline & $\begin{array}{c}\text { Mal der } \\
\text { Einspritzung }\end{array}$ & $\begin{array}{c}\text { Beziehung } \\
\text { zwischen der } \\
\text { Haupt-u. Mit- } \\
\text { agglutination }\end{array}$ & \multicolumn{2}{|c|}{ Beziehung zwischen der nachfolgenden } \\
\cline { 3 - 5 } & Igglutination
\end{tabular}

wurde. Dieser Wert fing wieder an abzunehmen, so dass er bei der neunten Immunisierung $\frac{1}{38}$ gross wurde (Tab. 4). Diese Schwankung wurde hier auch in einer Kurve auf die gleiche Weise dargestellt, wie bei den Paratyphus B-Bazillen (Kurve 2).

\section{Kurve 2.}

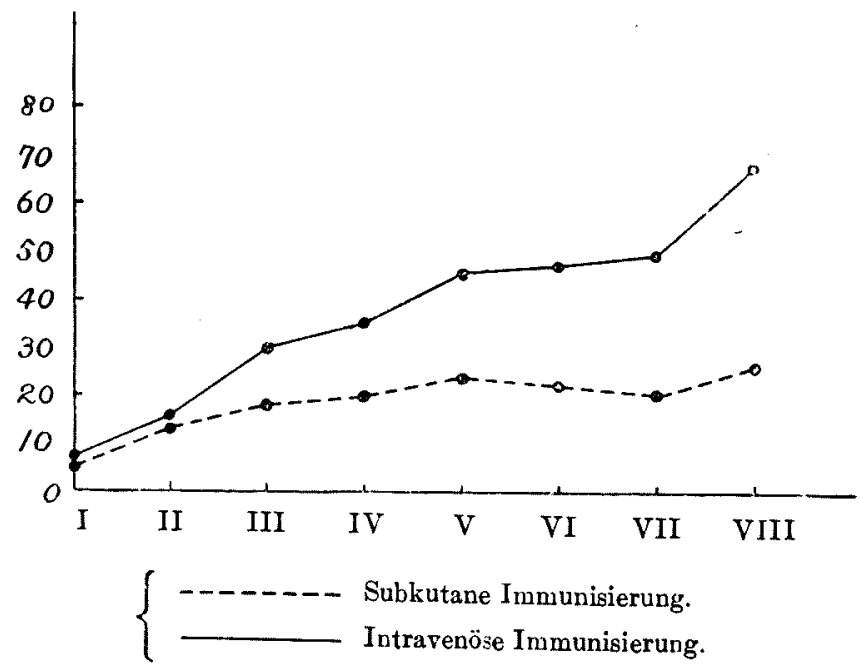




\section{Intraxenöse Immunisierung.}

Versuch 4.

Sechs Kaninchen wurden mit verschiedenen Dosen von sechs Stämmen Paratyphus A-Bazillen in siebentägigen Intervallen im ganzen siebenmal vorbehandelt. Die Dosen waren $\frac{1}{1000}, \frac{1}{500}, \frac{1}{200}, \frac{1}{100}, \frac{1}{50}, \frac{1}{20}, \frac{1}{10}, \frac{1}{5}, \frac{1}{2}$ und 1 Agarkultur. Am liebenten Tage nach jeder Vorbehandlung wurden Blutproben entnommen und auf ihren Agglutinationstiter geprüft. Darauf wurde der Titer der beiden Agglutinationen bei den einzeInen Tieren im Durchschnitt berechnet. Aus dieser Zahl wurde die Vermehrungsgeschwindigkeit der beiden Reaktionen und die Beziehung zwischen der Haupt- und Mitagglutination berechnet, wie schon oben auseinandergesetzt wurde.

\section{Versuch 5.}

Derselbe Versuch wurde mit noch grösseren Dosen derselben Bakterienstämme, nämlich $\frac{1}{1000}, \frac{1}{100}, \frac{1}{10}, \frac{1}{5}, \frac{1}{2}, 1,1 \frac{1}{2}$ u. $2 \frac{1}{2}$ Agarkulturen ausgeführt. Die Tiere wurden im ganzen achtmal vorbehandelt. Die Resultate wurden auf die gleiche Weise behandelt, wie beim vorigen Versuche.

\section{Versuch 6 .}

Hier wurde derselbe Versuch mit noch grösseren Disen Bakterien, nämlich $\frac{1}{100}, \frac{1}{10}, \frac{1}{5}, \frac{1}{2}, 1,1 \frac{1}{2}, 2$ u. $2 \frac{1}{2}$ Agarkulturen ausgeführt. Die Resultate wurden auf die gleiche Weise behandelt und beobachtet, wie bei den vorigen Versuchen.

\section{Versuch 7.}

Hier wurde wiederum derselbe Versuch nit noch grösseren Dosen Bakterien, nämlich $\frac{2}{10}, \frac{1}{3}, \frac{1}{2}, 1,1 \frac{x}{2}$ u. 2 Agarkulturen ausgeführt. Die Resultate wurden auf die gleiche Weise behandelt, wie bei den vorigen Versuchen.

Die Resultate aus den obigen vier Versuchen wurden je nach den Bakterienarten angeordnet und betrachtet, mit denen die Mitagglutination ausgeführt wurde. So wurden sie znerst nach Paratyphus B-Bazillen angeordnet. Auf diese Weise bekamen wir eine Tabelle. Aus dieser Tabelle, konnten wir die oben auseinandergesetzte Erscheinung zwar deutlich nachweisen. Der Deutlichkeit halber wurde aber eine durchschnittliche Zahl aus dieser Tabelle ansgerechnet. Das ergab Tabelle 5. Aus dieser Tabelle ist zu ersehen, dass die Vermehrungsgeschwindigkeit in den ersten vier Vorbehandlungen bei der Hauptagglutination bedeutend stärker als bei der Mitagglutination war, in der fünften bei der vorderen ebenso stark wie bei der letzteren, und in der sechsten bei der vorderen kleiner als bei der letzteren eintrat. Deshalb wurde die Schwankung des Wertes des Bruches, welche oben öter beschrieben worden ist, wïhrend der ganzen Vorbehandlungen deutlich nachgewiesen. Der Wert des Bruches nämlich, welcher in der ersten Vorbehandlung $\frac{\mathbf{1}}{\mathbf{2}}$ gross war, wurde mit den weiteren Vorbehand- 
Tabelle 5.

\begin{tabular}{|c|c|c|c|c|c|}
\hline \multirow{10}{*}{ 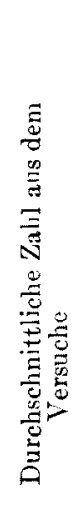 } & \multirow{2}{*}{$\begin{array}{l}\text { Mal der } \\
\text { Einspritzung }\end{array}$} & \multirow{2}{*}{$\begin{array}{l}\text { Beziehung } \\
\text { zwischen der } \\
\text { Haupt- u. Mit- } \\
\text { agglutination }\end{array}$} & \multicolumn{3}{|c|}{$\begin{array}{c}\text { Beziehung zwischen der nach folgenden } \\
\text { Agglutination }\end{array}$} \\
\hline & & & & Hauptagel. & Mitaggl. \\
\hline & & 197 & & & \\
\hline & 1 & $\begin{array}{l}5,404 \\
311\end{array}$ & $\mathrm{I}-\mathrm{II}$ & $\frac{23,4,58}{5,404}=4,3$ & $\frac{311}{197}=1,5$ \\
\hline & 11 & $\begin{array}{c}23,458 \\
463\end{array}=\frac{75}{1}$ & II-III & $\frac{64,291}{2458}=2,7$ & $\frac{413}{31}=1,5$ \\
\hline & III & $\overline{64,291}=\frac{1}{139}$ & $\mathrm{I}$ & $88,541=14$ & $387-08$ \\
\hline & IV & 357 & $111-1$ & $\overline{62,291}=1$ & $\overline{463}$ \\
\hline & & $\begin{array}{cc}88,541 & 228 \\
406 & 1\end{array}$ & $1 V-V$ & $\frac{93,749}{88,5+1}=1,0$ & $\frac{406}{387}=1,0$ \\
\hline & $\sqrt{ }$ & $\overline{93,749}=\overline{230}$ & & $94,999=1,0$ & $535=1,3$ \\
\hline & VI & $\frac{58 \%}{94,999}=\frac{1}{177}$ & & 93 & 406 \\
\hline
\end{tabular}

lungen immer kleiner, so dass er in der fünften Vorbehandlung $\frac{1}{230}$ gross sich zeigte. Dieser Wert wurde in der sechsten Immunisierung wieder grösser, nämlich $\frac{1}{177}$. Von der siebenten Immunisierung fing er an, wieder abzunehmen, so dass er bei der achten Vorbehandlung $\frac{1}{293}$ gross wurde. Dieses Verhalten kann man ganz deutlich aus Kurve 1 ersehen.

Als ferner die Resultate aus den letzten vier Versuchen nach Typhusbazillen angeordnet, mit denen die Mitagglutination ausgeführt worden war, betrachtet wurden, erhielten wir cine Tabelle. Der Deutlichkeit halber wurde eine durchschnittliche Zahl aus dieser Tabelle ausgerechnet. So bekamen wir Tabelle 6 . Daraus kann man folgendes ersehen: Die Vermehrungsgeschwindigkeit trat bei der Hauptagglutination in den ersten drei Vorbehandlungen nicht nur für sich allein, sondern auch im Vergleich mit der Mitagglutination bedeutend stärker, als bei der Mitagglutination ein, welche anch in dieser Zeit stärker als in der anderen zunahm. Und bei den weiteren Immunisierungen zeigte sie sich immer bei der Hauptagglutination stärker als bei der Mitagglutination. Da ferner der Titer bei der vorderen immer grösser als bei der letzteren war, wurde der Wert des Bruches, welcher in der ersten Immunisierung $\frac{1}{7}$ gross war, von der zweiten Vorbehandlung immer noch kleiner, so dass er sich in der achten Immunisierung am kleinsten während der ganzen Immunisierung nämlich $\frac{1}{67}$, darstellte, ohne dazwischen irgend eine Zunahme zu zeigen. Wenn man deshalb dieses Verhalteu in einer Kurve auf die gleiche Weise darstellt, so tritt die 
Tabelle 6.

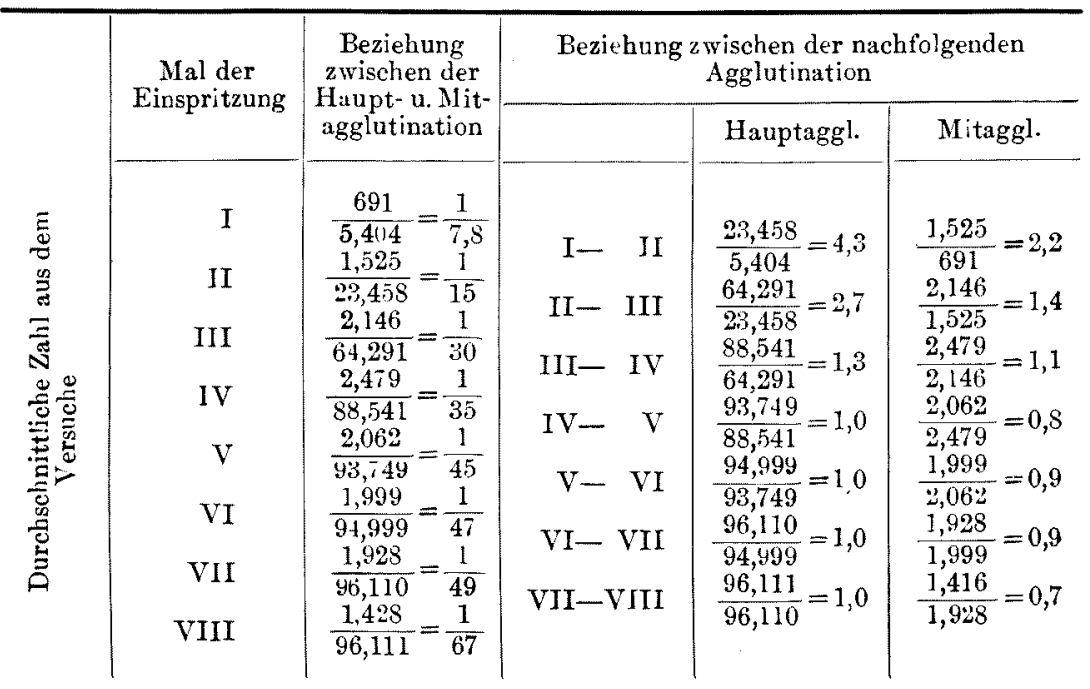

Kurvenlinie nicht so gekrümmt auf, wie bei der subkutanen Immunisierung (Kurve 2).

\section{Schlussbetrachtung.}

Wenn wir die einzelnen Betrachtungen vergleichend zusammenfassen, so ergibt sich folgendes:

Der Titer der Hauptagglutination hing auch bei Paratyphus A-Bazillen von der Dose der eingespritzten Bakterien und von der Immunisierungsmethode $a b$. Je grösser die eingespritzten Bakterien, desto höher trat ihr Titer ein. Dazu konnten wir bei intravenöser Vorbehandlung einen stärkeren Titer der Agglutination erhalten als bei sulkutaner Immunisierung, wie man gewöhnlich beobachten kann. Die Mitagglutination von Typhusbazillen und Paratyphus B-Bazillen, welche für sich allein kleiner als die Hauptagglutination war, verhielt sich in dieser Beziehung ganz gleich wie die Hauptagglutination. Was die Vermehrungsgeschwindigkeit anbelangt, so verhielt sie sich bei beiden Reaktionen nicht gleich. Bei der Hauptagglutination trat sie nämlich in einigen der ersten Immunisierungen nicht nur für sich allein, sondern auch im Vergleich zur Mitagglutination bedeutend stärker und in weiteren Vorbehandlungen ebenfalls kleiner als bei der Mitagglutination ein. Dagegen verhielt sie sich bei der Mitagglutina- 
tion ganz anders. So zeigte sie sich nämlich bei Paratyphus B-Bazillen im vorderen Stadium der Immunisierung nicht nur für sich allein, sondern auch im Vergleich mit der Hauptagglutination viel kleiner und in ihrem letzteren Stadium viel grösser als bei der Hauptagglutination. Deshalb verlief der Wert des Bruches bei diesen Bakterien in der ganzen Immunisierung so, dass er im Anfang der Vorbehandlung gross, dann kleiner und endlich wieder grösser wurde. Wenn die Immunisierung noch weiter fortgeführt wurde, so schien sich diese Schwankung nochmals $\mathrm{zu}$ wiederholen, wie wir schon in der dritten und achten Mitteilung angegeben hatten (Kurve 1). Bei Typhusbazillen war aber diese Erscheinung nicht immer bei allen Versuchen nachzuweisen. Wenn sie sich auch bei subkutaner Immunisierung noch deutlich erwies, so konnten wir siè doch bei intravenöser Vorbehandlung nicht nachweisen, weil die Vermehrungsgeschwindigkeit der Mitagglutination bei der ganzen Immunisierung im Vergleich mit der der Hauptagglutination immer kleiner war. Da ferner der Titer der Hauptagglutination immer grösser als der Titer der Mitagglutination eintrat, verlief der Wert des Bruches in der ganzen Immunisierung so, dass er mit der Immunisierung fortschreitend immer kleiner wurde, ohne dazwischen eine Zunahme zu zeigen (Kurve 2). Ein weiterer Unterschied, welcher zwischen den beiden Bakterienarten Immunserum von Paratyphus ABazillen gegenüber nachzuweisen war, bestand darin, dass die Mitagglutination bei Typhusbazillen viel stärker als bei Paratyphus B-Bazillen eintrat. Deshalb fanden wir den Wert des Bruches bei den vorderen Bakterien immer grösser als bei den letzteren, wie man aus den Tabellen deutlich ersehen kann. Nach obigen Ergebnissen können wir annehmen, dass die Erscheinung, welche in der Einleitung kurz auseinandergesetzt wurde, bei der Immunisierung mit Paratyphus A-Bazillen Paratyphus B-Bazillen gegenüber sowohl bei subkutaner Vorbehandlung als auch bei intravenöser ganz deutlich nachweisbar ist. Was Typhusbazillen anbelangt, trat sie zwar bei subliutaner Vorbehandlung ziemlich deutlich, aber bei intravenöser Immunisierung so undeutlich auf, dass die Kurvenlinie fast nicht gekrümmt, sondern mehr gerade verlief.

Wenn man noch andere Erscheinungen auf Grund dieser Ergebuisse mit anderen, welche in früheren Mitteilungen angegeben worden sind, vergleicht, so ist folgendes hervorzuheben:

1. Der Zeitpunkt, wo der kleinste Wert des Bruches in der ersten Schwankung nachweisbar war, schien auch hier bei boiden Bakterienar- 
ten sowohl bei subkutaner Imınunisierung als auch bei intravenöser bei einer kleineren Dose später als bei einer grösseren einzutreten.

2. Aber die Erscheinung, dass der Wert des Bruches bei der Immunisierung mit kleiner Dose sich kleiner als bei der mit grösserer zeigte, war hier nicht deutlich nachzuweisen.

3. Ebenso war eine noch andere Erscheinung, dass der Wert des Bruches bei intravenöser Immunisierung grösser als bei subkutaner Vorbehandlung eintrat, wie schon in der ersten, dritten und achten Mitteilung angegeben wurde, hier bei beiden Bakterienarten nicht nachzuweisen. Vielmehr trat er bei der vorderer Immunisierung kleiner als bei letzterer auf.

4. Zum Schluss möchten wir noch eine andere Erscheinung hier herrorheben, welche bis jetzt noch nicht beobachtet wurde. Sie bestand nämlich darin, dass der kleinste Wert des Bruches sich in der ersten Schwankung viel grösser als in der zweiten zeigte. Und dazu trat er bei Paratyphus B-Bazillen so klein auf, wie wir ihn in den früheren Mitteilungen nicht finden konnten. Auf die gleiche Weise stellte sich der grösste Wert des Bruches, welcher am Ende der ersten Schwankung eintrat, niemals so gross dar wie im Anfang derselben, sondern er blieb so klein, dass man zwischen ihm und dem anderen, nämlich zwischen dem grössten und kleinsten Wert des Bruches, keinen grossen Unterschied finden konnte. Dieser Unterschied war besonders bei Typhusbazillen kaum nachzuweisen. Die Kurvenlinie, welche den Verlauf des Wertes des Bruches in der ganzen Immunisierung darstellt, verlief deshalb bei Paratyphus B-Bazillen stärlker gekrümmt, aber immer weit von der Horizontallinie und dagegen bei Typhusbazillen weniger gekrümmt, aber wenig von der Horizontallinie entfernt. Dieses ganze Verhalten kann man ganz deutlich ausfindig machen, wenn man die Kurven, welche oben angegeben wurden, mit den anderen, welche in den früheren Mitteilungen, nämlich in der ersten, dritten und achten, genau dargestellt worden sind, vergleichend betrachtet.

\section{Literatur.}

(1) Aoki u. Konno, Über die Beziehung zwischen der Haupt- und Milagglutination, J. Mitteilung. Centralbl. f. Bakt. 1. Abt. Orig. 1920, 86, 139.

(2) A oki u. Konno, III. Mitteilung. Tohoku Journ. of Exp. Med. 1920, 1, 475.

(3) Aoki u. Konno, VI. Mitteilung. Tohoku Journ. of Exp. Med. $19212,75$.

(4) A oki u. Konno, VIII. Mitteilung. Tohoku Journ. of Exp. Med. 1921, 2, 376. 